\title{
Laryngeal Fistula, CTCAE
}

National Cancer Institute

\section{Source}

National Cancer Institute. Laryngeal Fistula, CT CAE. NCI Thesaurus. Code C58190.

A disorder characterized by an abnormal communication between the larynx and another organ or anatomic site. 